OPEN ACCESS

Check for updates

\section{Association between characteristics of behavioural weight loss programmes and weight change after programme end: systematic review and meta-analysis}

\author{
Jamie Hartmann-Boyce, ${ }^{1}$ Annika Theodoulou, ${ }^{1}$ Jason L Oke, ${ }^{2}$ Ailsa R Butler, ${ }^{1}$ \\ Peter Scarborough, ${ }^{3}$ Anastasios Bastounis, ${ }^{1,4}$ Anna Dunnigan, ${ }^{5}$ Rimu Byadya,,${ }^{6,7}$ \\ F D Richard Hobbs, ${ }^{1}$ Falko F Sniehotta, ${ }^{8,9}$ Susan A Jebb, ${ }^{1}$ Paul Aveyard ${ }^{1}$
}

For numbered affiliations see end of the article

Correspondence to:

J Hartmann-Boyce

jamie.hartmann-boyce@phc.

ox.ac.uk

(or @jhb19 on Twitter

ORCID 0000-0001-9898-3049)

Additional material is published online only. To view please visit the journal online.

Cite this as: $B M J$ J 2021;374:n1840 http://dx.doi.org/10.1136/bmj.n1840

Accepted: 15 July 2021

\begin{abstract}
OBJECTIVE

To determine if the characteristics of behavioural weight loss programmes influence the rate of change in weight after the end of the programme.

DESIGN

Systematic review and meta-analysis.

DATA SOURCES

Trial registries, 11 electronic databases, and forward citation searching (from database inception; latest search December 2019). Randomised trials of behavioural weight loss programmes in adults with overweight or obesity, reporting outcomes at $\geq 12$ months, including at the end of the programme and after the end of the programme.
\end{abstract}

\section{REVIEW METHODS}

Studies were screened by two independent reviewers with discrepancies resolved by discussion. $5 \%$ of the studies identified in the searches met the inclusion criteria. One reviewer extracted the data and a second reviewer checked the data. Risk of bias was assessed with Cochrane's risk of bias tool (version 1). The rate of change in weight was calculated $(\mathrm{kg} / \mathrm{month}$; converted to $\mathrm{kg} /$ year for interpretability) after the end of the programme in the intervention versus control groups by a mixed model with a random intercept. Associations between the rate of change in weight and prespecified variables were tested.

RESULTS

Data were analysed from 249 trials $(n=59081)$ with a mean length of follow-up of two years (longest 30

\section{WHAT IS ALREADY KNOWN ON THIS TOPIC}

Behavioural weight management programmes are recommended for the treatment of obesity in adults

Most behavioural weight loss programmes produce short term losses in weight but the rate of change in weight after the end of the programme is highly variable and the causes of this variation are largely unknown

\section{WHAT THIS STUDY ADDS}

Greater amounts of weight lost during the programme and financial incentives for weight loss were significantly associated with greater regain in weight after the end of the programme in all models, but greater initial weight loss was still associated with added benefits for at least five years after the end of the programme

Access to the behavioural weight loss programme by participants outside of the study was the only variable associated with slower regain in weight

Few studies provided data at or beyond five years and therefore the results should not be extrapolated beyond this point years). $56 \%$ of studies ( $n=140$ ) had an unclear risk of bias, $21 \%(n=52)$ a low risk, and $23 \%(n=57)$ a high risk of bias. Regain in weight was faster in the intervention versus the no intervention control groups (0.12-0.32 kg/year) but the difference between groups was maintained for at least five years. Each kilogram of weight lost at the end of the programme was associated with faster regain in weight at a rate of $0.13-0.19 \mathrm{~kg} /$ year. Financial incentives for weight loss were associated with faster regain in weight at a rate of $1-1.5 \mathrm{~kg} /$ year. Compared with programmes with no meal replacements, interventions involving partial meal replacements were associated with faster regain in weight but not after adjustment for weight loss during the programme. Access to the programme outside of the study was associated with slower regain in weight. Programmes where the intensity of the interaction reduced gradually were also associated with slower regain in weight in the multivariable analysis, although the point estimate suggested that the association was small. Other characteristics did not explain the heterogeneity in regain in weight.

\section{CONCLUSION}

Faster regain in weight after weight loss was associated with greater initial weight loss, but greater initial weight loss was still associated with reduced weight for at least five years after the end of the programme, after which data were limited. Continued availability of the programme to participants outside of the study predicted a slower regain in weight, and provision of financial incentives predicted faster regain in weight; no other clear associations were found.

STUDY REGISTRATION

PROSPERO CRD42018105744.

\section{Introduction}

Excess adiposity is a leading cause of preventable disease and death worldwide. ${ }^{1}$ Behavioural weight management programmes that aim to achieve weight loss in adults by changes in diet, exercise, or both, are recommended for the treatment of obesity. Most programmes produce short term weight loss but considerable uncertainty exists about their longer term effects.

In a companion systematic review, pooled analyses from 145 studies comparing behavioural weight loss programmes with minimal intervention controls estimated that regain in weight after the end of the programme was $0.12-0.32 \mathrm{~kg} /$ year in intervention groups 
relative to a minimal or no intervention comparator, with a difference between groups evident for at least five years but with considerable variation in the rate of regain in weight (unpublished data). Behavioural weight loss programmes reduced the incidence of type 2 diabetes and hypertension, with reductions in risk factors for cardiovascular disease and improvements in quality of life corresponding to the weight loss. Regain in weight reversed some of these differences, however, with improvements in blood pressure, lipids, and quality of life seen at the end of the programme declining over time in association with regain in weight.

Concern about regain in weight and the long term value of attempts at weight loss has been identified as a barrier to engagement in behavioural weight loss programmes from patients and healthcare providers. ${ }^{2}$ Considerable research has been done on developing and testing specific weight loss maintenance programmes, but with little success. ${ }^{34}$ Programmes have achieved modest success, with behavioural support intended to promote lasting adherence to changes in diet and increased physical activity. ${ }^{5}$ Continuing support is expensive, however, and most people choose not to take it up. ${ }^{6}$

Our study aimed to determine whether specific characteristics of behavioural weight loss programmes influence the rate of change in weight after the end of the programme, specifically focusing on the effect of these programmes on regain in weight. Such evidence could inform the development of interventions, patient choice, and expectations of practitioners, and improve the effectiveness of treatment in the long term for people with overweight or obesity.

\section{Methods}

A protocol was registered in advance; full methodological details are in the protocol ${ }^{7}$ and in a companion publication (unpublished).

\section{Data sources and searches}

We searched for randomised controlled trials in clinical trials registries (clinicaltrials.gov/ and www. isrctn.com/) and 11 electronic databases, with terms relating to obesity, weight loss, diet, exercise, behaviour change, and behavioural weight loss programmes. We also searched the Health Services Research Unit's (University of Aberdeen) register of weight loss trials. Searches were run from database inception to September 2018 and were restricted to full papers published in English. The authors were contacted for additional information where necessary. In December 2019 (before the analyses), we conducted forward citation searches for included or ongoing studies where the most recent publication was from January 2007 onwards, to identify additional longer term follow-up data.

\section{Study selection}

Two reviewers independently screened studies for inclusion, with disagreements resolved by discussion or by referral to a third reviewer. We included randomised controlled trials of non-pregnant adults (aged $\geq 18$ ) who were mostly with overweight or obesity (body mass index $\geq 25$, or $\geq 23$ in Asian populations) at the start of the study. Interventions included behavioural weight loss programmes delivered in any setting, and comparators were another behavioural weight loss programme, a less intensive intervention, or no intervention. We excluded interventions targeting multiple risk factors (eg, also including interventions for stopping smoking) and interventions involving drug treatments, surgery, or both. Studies had to follow participants for $\geq 12$ months from baseline and measure the change in weight at the end of the programme and after the end of the programme. We defined the end of the programme as the time when the intensity of the contact was considerably reduced or ended (full details are in the protocol ${ }^{7}$ ).

\section{Outcome}

Our primary outcome was rate of change in weight after the end of the programme in the intervention group relative to the control groups. In most of the studies included in the review, data on weight were based on complete case or multiple imputation methods. The control group was determined by the intensity of the intervention (that is, comparing the most intensive with the least intensive intervention). This definition was based on contact time and the elements of the programme (eg, in a study of behavioural support with meal replacements $v$ behavioural support only, the meal replacement arm would be the primary intervention of interest).

\section{Variables}

We were interested in whether the rate of change in weight (regain) after the end of the programme was associated with characteristics of the behavioural weight loss programme, identified a priori. Initially, we had also planned to investigate if the rate of regain in weight was associated with whether involvement with the programme was self-initiated, prompted, or required, and whether the programme was tailored, but insufficient data were available to support these analyses.

The characteristics that we investigated include:

- Meal replacements (eg, a portion controlled drink, bar, or soup intended as a substitute for a traditional meal), categorised as total meal replacements (often but not always very low energy diets), partial meal replacements, or no meal replacements

- Strategies to change diet, physical activity, or both

- The programme includes support for maintaining weight loss (defined as content designed to help participants maintain their weight loss after the end of the programme (eg, relapse prevention or weight maintenance components))

- $\quad$ Reducing the intensity of the intervention during the programme (defined as a change in the frequency of contact with the intervention)

- Inpatient or other residential care 
- Financial incentives (where financial rewards depend on achieving weight loss)

- Periods of fasting (eg, eating that is time restricted, including but not limited to programmes such as $5: 2$, alternate day fasting, time restricted eating, and prolonged fasting)

- Whether the programme was publicly available outside of the study context

- $\quad$ Length of intervention (months).

- Outcomes of the behavioural weight loss programme include:

- Difference between the intervention and control group in weight $(\mathrm{kg})$ at the end of the programme; of note, this differencealso indirectly takes into account the variation in intensity in the control group

- $\quad$ Rate of weight loss during the programme (kg lost/year, intervention $v$ control group).

\section{Data extraction and quality assessment}

Data extraction was conducted with a database with bespoke fields for this review, piloted by four reviewers before its use. We assessed the risk of bias at the study level in: random sequence generation; allocation concealment; blinding of outcome assessment; attrition; and other risk of bias with the Cochrane risk of bias tool (version 1) for randomised trials. ${ }^{8}$

Data extraction and assessments of risk of bias were conducted by one reviewer and checked by a second reviewer. Discrepancies were resolved by discussion or by referral to a third reviewer.

\section{Data synthesis and analysis}

We calculated the difference in weight change between arms at all available time points. With a mixed model with a random intercept for each study, we regressed difference in weight between arms on time from the end of the programme to calculate the rate of regain in weight in kilograms per month in the intervention group relative to the control groups. In this three level model, measurements of regain in weight at different times were nested within arms, nested within studies. Some trials tested behavioural weight loss programmes against one another and others tested behavioural weight loss programmes against no intervention or a minimal intervention, which might contribute to heterogeneity. To reflect these differences, we adjusted for difference in weight between arms at the end of the programme. In the post hoc sensitivity analyses, we added a variable to indicate that the comparator was no intervention or minimal intervention and the results were almost identical.

We tested for associations between our prespecified variables and the rate of regain in weight in a univariable model. These models included a term for the presence of that variable and variable $\times$ time. We then tested associations with three multivariable approaches: (A) a model including only programme characteristics, with a backwards selection model; (B) a model including only weight variables (rate of weight loss and difference in weight at the end of the programme); and (C) a combined model including weight variables and variables retained in model $\mathrm{A}$.

We used $\mathrm{P}>0.05$ (that is, 95\% confidence intervals excluding the null) as a nominal threshold for determining statistical significance and which variables to retain in the models. In these analyses, programmes with characteristics that were associated with slower regain in weight would have negative signs.

Planned sensitivity analyses excluded studies at high risk of bias in any domain. Post hoc sensitivity analyses also removed studies with a high and unclear risk of bias (eg, including only studies at low risk of bias). To investigate possible publication bias, we assessed whether the length of follow-up was associated with the amount of weight lost at the end of the programme with Pearson's correlation coefficient. If a study had not found a difference in weight at the end of the programme, it might be less likely to follow up participants after the end of the programme because investigators might not want to use or obtain additional resources to conduct the follow-up. All analyses were carried out in $\mathrm{R}$ version 4.0.2.

\section{Patient and public involvement}

This project emerged out of feedback from a panel of members of the public with lived experience of obesity. Two patient and public involvement advisors shaped the research before the protocol was finalised; in particular, they prioritised which programme characteristics to focus on in our analyses. They are also supporting dissemination of the results.

\section{Results}

\section{Search results}

Our initial searches retrieved 17085 references, 4482 of which were screened for full text. The most common reason for exclusion after review of the full text was short length of follow-up (eFigure 1). Another 246 relevant references were identified by forward citation searching and screening of trial websites. A total of 879 references representing 330 studies met our inclusion criteria ( $5 \%$ of the studies initially identified). The authors of 53 included studies provided more information. Our analyses included 249 studies $(n=59081)$ that provided sufficient information (eTable 1 ).

\section{Characteristics of included studies}

Table 1 shows summary data for the studies included in the systematic review. eTable 1 (primary references), eTable 2 (assessments of risk of bias), eTable 3 (key characteristics), eTable 4 (baseline demographics), and eTable 5 (characteristics of the intervention) provide details of individual studies. Seven studies followed up participants for five years or more $(n=8149)$.

\section{Risk of bias}

The risk of bias was unclear in 56\% of studies, mainly because they did not fully report the randomisation procedures, low in 21\%, and high in 23\% (table 1). eTable 2 describes assessments of the risk of bias for each study. 
Change in weight after the end of the programme

As will be reported in detail elsewhere but reiterated here to provide context, at the end of the programme, the average change in weight in the minimal intervention (control) arms was $-2.1 \mathrm{~kg}$ (standard deviation 3.3) and in the intervention arms was -4.9 $\mathrm{kg}$ (3.8), a mean difference in weight in favour of the interventions of $-2.8 \mathrm{~kg}$ (95\% confidence interval -3.2 to -2.4 ; unpublished data). In the 145 studies that compared behavioural weight loss programmes with minimal intervention and in which participants receiving the behavioural weight loss programme lost weight relative to controls at the end of the programme, regain in weight was faster in the intervention versus the no intervention control groups $(0.12-0.32 \mathrm{~kg} /$ year). We found a difference in weight in favour of the intervention group for at least five years, although beyond five years the data were limited.

\section{Association between programme variables and subsequent regain in weight}

In univariable analysis, financial incentives for weight loss and provision of partial meal replacements were strongly associated with faster regain in weight (table 2). Regain in weight was also more rapid in programmes that included advice to exercise or opportunities for exercise, or that advised fasting, but these associations were weaker and not statistically significant (table 2). We found no meaningful association between length of programme and regain in weight. Availability of the programme to participants outside of the trial was associated with significantly slower regain in weight in univariable and multivariable models (table 2). Regain in weight was also slower in programmes that advised changes in diet, provided strategies to support maintenance of weight loss, provided an inpatient or residential phase of treatment, or where the intensity

\begin{tabular}{|c|c|}
\hline Characteristic & No, median (IQR), or mean (range) \\
\hline \multicolumn{2}{|l|}{ Geographical region (No of studies $(n=249)$ ) } \\
\hline North America & 145 \\
\hline South America & 2 \\
\hline Europe & 73 \\
\hline Asia & 10 \\
\hline Australia and New Zealand & 17 \\
\hline Africa & 1 \\
\hline Mixed (Australia and Europe) & 1 \\
\hline \multicolumn{2}{|l|}{ Meal replacements (No of arms $(n=629)$ ) } \\
\hline Partial & 45 \\
\hline Total & 29 \\
\hline \multicolumn{2}{|l|}{ Diet or exercise (No of arms $(n=629))$} \\
\hline Diet and exercise & 382 \\
\hline Diet only & 126 \\
\hline Exercise only & 20 \\
\hline \multicolumn{2}{|c|}{ Intervention included content designed to help participants after end of programme (No of arms ( $\mathrm{n}=629)$ ) } \\
\hline Yes & 217 \\
\hline No & 397 \\
\hline Not reported & 15 \\
\hline \multicolumn{2}{|l|}{ Reducing intensity of intervention during the programme (No of arms $(n=629)$ ) } \\
\hline Yes & 250 \\
\hline No & 365 \\
\hline Not reported & 14 \\
\hline \multicolumn{2}{|l|}{ Inpatient or other residential care (No of arms $(n=629)$ ) } \\
\hline Inpatient & 18 \\
\hline Residential & 2 \\
\hline Financial incentives for weight loss (No of arms $(n=629)$ ) & 31 \\
\hline Periods of fasting (No of arms $(\mathrm{n}=629))$ & 6 \\
\hline \multicolumn{2}{|l|}{ Programme was publicly available outside of the study context (No of arms $(n=629)$ ) } \\
\hline Yes & 51 \\
\hline No & 563 \\
\hline Not reported & 15 \\
\hline Age (median (IQR)) & 48.2 (11.0); $n=519$ study arms \\
\hline Baseline body mass index (median (IQR)) & $33.7(4.7) ; n=462$ study arms \\
\hline \multicolumn{2}{|l|}{ Programme length (months; mean (range)) } \\
\hline Follow-up after end of programme & 23.4 (11.5-360; median 11.85) \\
\hline Most intensive intervention arm & $6.5(0.7-72)$ \\
\hline Longest study arm & $6.9(0.7-72)$ \\
\hline \multicolumn{2}{|l|}{ Assessment of risk of bias (low/unclear/high) (No of studies) } \\
\hline Overall risk of bias & $52 / 140 / 57$ \\
\hline Selection bias (random sequence generation and allocation concealment) & $76 / 171 / 2$ \\
\hline Detection bias & $214 / 26 / 9$ \\
\hline Attrition bias & $205 / 11 / 33$ \\
\hline Other risk of bias (only assessed where suspected) & $\mathrm{NA} / \mathrm{NA} / 20$ \\
\hline
\end{tabular}


of contact with the intervention was reduced through the programme but the associations were weaker and not statistically significant. Table 2 shows all associations; statistically significant associations are illustrated in figure 1, with studies divided into those with and without the characteristic, and regression lines fitted through each trial arm estimate. Figure 1 also shows the effect of interventions which reduced in intensity over time; this variable emerged as a statistically significant association in the model adjusted for weight loss during the programme (table 3). The multivariable analysis used backwards selection to remove any variable not significantly associated with regain in weight and produced estimates that were similar to those of the univariable analysis (table 2).

In sensitivity analyses (eTables 6 and 7), the variable, intervention reducing in intensity over time, was no longer retained, and inpatient setting emerged in its place (no significant association was found for either). Partial meal replacement remained a statistically significant predictor of faster regain in weight, but similar to the univariate analyses, the association was stronger when studies at high risk of bias were removed, with confidence intervals excluding the original estimate $(0.134 \mathrm{~kg} / \mathrm{month}$ (95\% confidence interval 0.071 to 0.196$) v 0.037 \mathrm{~kg} /$ month). Total meal replacement was a statistically significant predictor of faster regain in weight when studies at high risk of bias were removed $(0.074 \mathrm{~kg} /$ month, 95\% confidence interval 0.022 to 0.126 ) (eTable 8). Estimates for financial incentives and availability of the programme to participants outside of the study did not meaningfully change when studies at high risk of bias were removed. In our post hoc sensitivity analysis studies assessed as being at low risk of bias, we included the same variables as in the backwards selection model and found no significant differences in estimates, with confidence intervals overlapping those in the primary model in all instances.

\section{Change in weight during the programme}

Greater and faster weight loss during the weight loss programme were associated with faster regain in weight. In univariable analysis, for each kilogram of weight loss achieved at the end of the programme, regain in weight occurred 0.011 (95\% confidence interval 0.0085 to 0.014$) \mathrm{kg} / \mathrm{month}$ faster. Also, more rapid weight loss during the programme was associated with faster regain in weight; the estimate for regain in weight after the programme was 0.03 (0.02 to 0.041) $\mathrm{kg} /$ year faster for each $\mathrm{kg} /$ year faster weight loss during the programme (estimates were converted to years for ease of interpretation). In multivariable analysis, the estimate for weight loss by the end of the programme was similar at $0.012(0.0080$ to 0.016$) \mathrm{kg} / \mathrm{month}$, but more rapid weight loss was close to the null $(-0.0053$ $(-0.021$ to 0.010$) \mathrm{kg} / \mathrm{month})$. Figure 2 illustrates the association between weight loss achieved during the programme and regain in weight after the programme, dividing the trial arms by achieved weight loss at the end of the programme relative to controls, and fitting regression lines through the estimates.

In planned sensitivity analyses (eTables 6 and 8), the direction of the association did not change and confidence intervals overlapped, with the exception of change in weight at the end of the programme in the multivariable model including rate of weight loss and change in weight at programme end, where the trend estimate was steeper and the confidence intervals did not overlap with those in the main analysis (0.026 $\mathrm{kg} /$ month (95\% confidence interval 0.018 to 0.034 ) $v 0.012 \mathrm{~kg} / \mathrm{month})$. In post hoc sensitivity analyses including only studies at low risk of bias, the results were consistent with those from the main models for the univariable and multivariable analyses.

\section{Association between programme variables and subsequent regain in weight adjusted for weight loss during the programme}

Adjusting for the extent and rate of weight loss during the programme markedly changed the estimates

\begin{tabular}{|c|c|c|}
\hline Characteristic & \multicolumn{2}{|c|}{ Trend estimate $(\mathrm{kg} /$ month; $95 \% \mathrm{Cl})$} \\
\hline Meal replacements (partial) & $0.035(0.015 \text { to } 0.055)^{\star}$ & $0.037(0.017 \text { to } 0.057)^{\star}$ \\
\hline Meal replacements (total) & $0.014(-0.013$ to 0.041$)$ & $0.018(-0.0089$ to 0.044$) \neq$ \\
\hline Programme included exercise & $0.013(-0.0073$ to 0.034$)$ & - \\
\hline Programme included support for weight maintenance & $-0.0062(-0.024$ to 0.012$)$ & - \\
\hline Reducing intensity of intervention & $-0.0085(-0.032$ to 0.016$)$ & $-0.022(-0.046$ to 0.0017$) \S$ \\
\hline Intervention inpatient & $-0.072(-0.22$ to 0.082$)$ & - \\
\hline Intervention other residential & $-0.0052(-0.095$ to 0.085$)$ & - \\
\hline Financial incentives for weight loss & $0.13(0.043 \text { to } 0.21)^{\star}$ & $0.13(0.046 \text { to } 0.21)^{*}$ \\
\hline
\end{tabular}



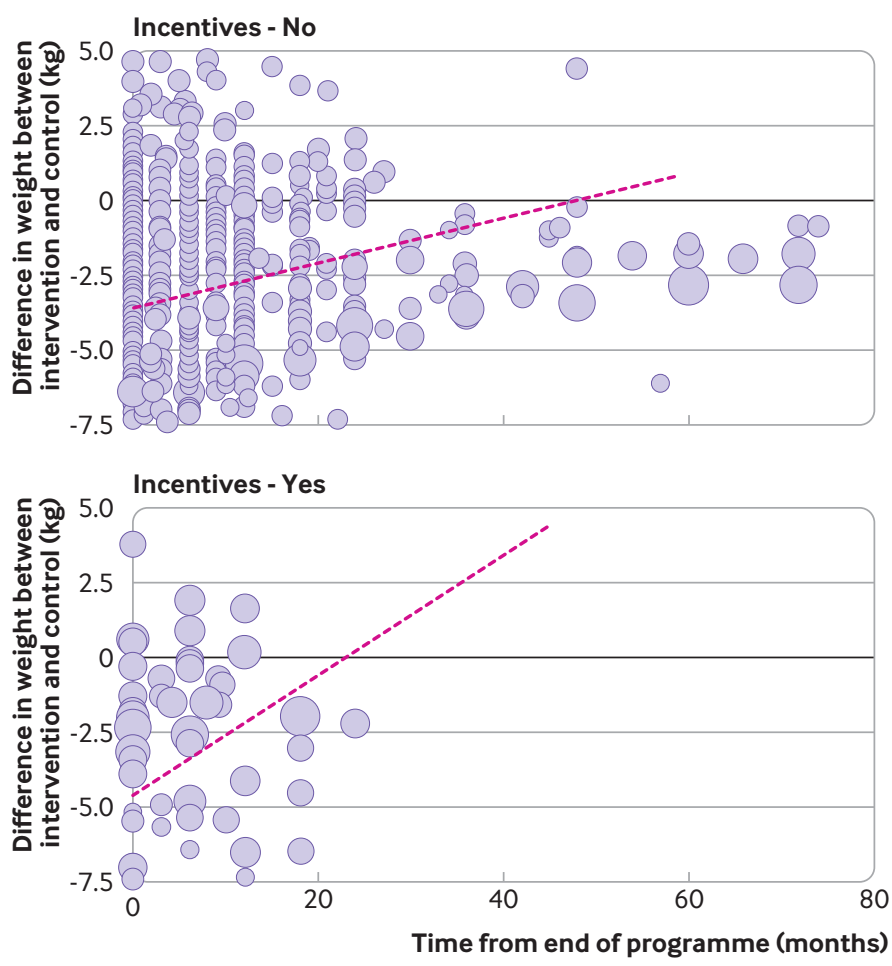

Fig 1 | Rate of change in weight after end of behavioural weight management programme, by selected characteristics of programmes

for meal replacement programmes (table 3). After adjustment, the point estimates suggested that meal replacement programmes were associated with slower regain in weight but were close to the null and not significant.

Programmes where the intensity of the interaction declined gradually over time were statistically significantly associated with slower regain in weight in the multivariable analysis (table 3), which was not the case in the univariable analysis, although the point estimate suggested that the association was small. The association between financial incentives and more rapid regain in weight was reduced by $30 \%$ when adjusted for weight loss during the programme, and the slower regain in weight associated with the availability of the programme outside of the trial was reduced by $8 \%$.

In planned sensitivity analyses removing studies at high risk of bias, partial meal replacements re-emerged as statistically significantly associated with faster regain in weight $(0.11 \mathrm{~kg} / \mathrm{month}, 95 \%$ confidence interval 0.049 to 0.161 ). Planned sensitivity analyses did not substantially affect estimates for any of the other variables in multivariable model (eTable 9). In post hoc analyses including only studies at low risk of bias, estimates were not substantially altered for any variables (consistent direction of association and overlapping confidence intervals in all instances).

\section{Discussion \\ Principal findings}

Our meta-analysis of 249 studies of behavioural weight loss programmes identified substantial variation in regain in weight after the end of the programme. In our analyses, we consistently found that initial weight loss was associated with later regain in weight, with every additional kilogram of weight lost in the intervention group relative to the comparator group during the programme associated with faster regain in weight at a rate of $0.13-0.19 \mathrm{~kg} /$ year after the end of the programme. Programmes providing financial incentives for weight loss were independently associated with faster regain in weight of about $1 \mathrm{~kg}$ /year. Programmes providing partial meal replacement products were associated with faster regain in weight; in our main model, this finding was explained by weight loss achieved in the programme, but in sensitivity analyses where studies at high risk of bias were removed, partial meal replacements remained independently associated with greater regain in weight. In contrast, the availability of the behavioural weight loss programme to participants outside of the study protocol was associated with substantially less regain in weight ( $3 \mathrm{~kg} /$ year). Some evidence indicated that programmes where the intensity of the interventions was reduced over time were also associated with slower regain in weight after the end of the programme (about $0.3 \mathrm{~kg}$ slower per year).

\begin{tabular}{|c|c|c|}
\hline \multirow[b]{2}{*}{ Characteristic } & \multicolumn{2}{|l|}{ Trend estimate (kg/month; $95 \% \mathrm{Cl})$} \\
\hline & $\begin{array}{l}\text { Multivariable models A or B (programme } \\
\text { characteristics or weight lost during programme) }\end{array}$ & $\begin{array}{l}\text { Multivariable model C (weight and } \\
\text { programme characteristics) }\end{array}$ \\
\hline \multicolumn{3}{|l|}{ Programme characteristics } \\
\hline Partial meal replacements & $0.037(-2.6 \text { to }-0.35)^{\star}$ & $-0.022(-0.049$ to 0.0041$)$ \\
\hline Total meal replacements & $0.018(-0.0089$ to 0.044$)$ & $-0.015(-0.041$ to 0.011$)$ \\
\hline $\begin{array}{l}\text { Reducing intensity of intervention } \\
\text { during programme }\end{array}$ & $-0.022(-0.046$ to 0.0017$)$ & $-0.026(-0.047 \text { to }-0.0046)^{\star}$ \\
\hline Financial incentives for weight loss & $0.13(0.046 \text { to } 0.21)^{\star}$ & $0.090(0.020 \text { to } 0.16)^{*}$ \\
\hline Programme available outside study & $-0.26(-0.37 \text { to }-0.14)^{\star}$ & $-0.24(-0.35 \text { to }-0.14)^{\star}$ \\
\hline \multicolumn{3}{|l|}{ Weight lost during programme } \\
\hline $\begin{array}{l}\text { Weight difference at end of } \\
\text { programme }(\mathrm{kg})\end{array}$ & $0.012(0.0080 \text { to } 0.016)^{\star}$ & $0.016(0.010 \text { to } 0.022)^{\star}$ \\
\hline $\begin{array}{l}\text { Rate of weight loss during programme } \\
\text { (kg lost/year) }\end{array}$ & $-0.0053(-0.021$ to 0.010$)$ & $-0.013(-0.032$ to 0.0052$)$ \\
\hline
\end{tabular}



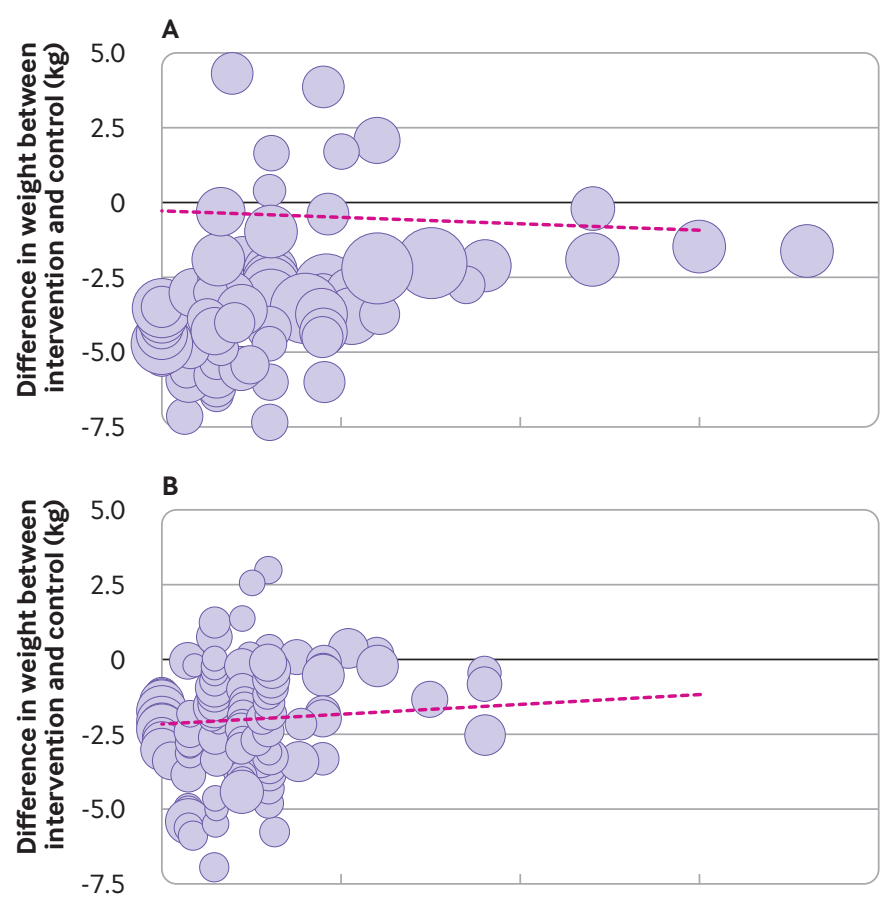

$-7.5$



Fig 2 | Rate of change in weight after end of behavioural weight management programme, by weight loss during the programme. Difference in weight at end of programme between intervention and control groups: $A$, up to $-1.5 \mathrm{~kg} ; \mathrm{B},-1.4$ to -3 $\mathrm{kg}$;,-3.1 to $-5 \mathrm{~kg}$. Graphs are illustrative only and analyses are based on mixed models. Lines are based on changes within trials, and because the points mapped represent different trials, the lines will not necessarily be visually consistent with the mapped points. In sensitivity analyses, removing studies at high risk of bias (eTable 6), univariate analyses were not substantially different with the exception that the association between partial meal replacements and regain in weight was stronger, with confidence intervals excluding the original estimate $(0.15 \mathrm{~kg} / \mathrm{month}$ ( $95 \%$ confidence interval 0.087 to 0.21$) v 0.035 \mathrm{~kg} /$ month). In a post hoc analysis, a similar pattern was found when only studies at low risk of bias were included; univariate analyses were significantly different only for partial meal replacements where the point estimate and confidence intervals were the same as in the sensitivity analysis including studies at low and unclear risk of bias
0.03 body mass index unit per month (about $1 \mathrm{~kg} /$ year) after the end of the active programmes, and found no associations between the content of the programme and slopes for changes in weight more than 12 months from baseline. We found much lower rates of regain in weight but these rates were relative to the randomised comparator rather than absolute values, and were based on many more studies and larger sample sizes, with longer term data, providing greater confidence in the new estimates.

Our review had some limitations. Because of the lack of uniform standards for reporting of weight loss outcomes, we made several assumptions in coding and analysing our data. Our focus on relative differences between the intervention and comparator should not have been unduly influenced by these assumption. We coded the content of the intervention according to a preestablished framework described in the protocol but we were limited by the level of detail available. Almost a quarter of the studies included in our review were assessed as being at high risk of bias; removing these studies did not affect most of our estimates but led to uncertainty about the estimates for meal replacements. We relied on statistical significance to select variables for subsequent modelling; this approach is common but could be prone to errors because of multiple testing. Our test for publication bias was not significant (unpublished data), but we cannot completely rule it out. Our latest search date was December 2019 and studies published subsequently were missed; however, we contacted the authors of unpublished studies and hence some of these data were available to us before publication. Because of the size of the review, a time lag between searches and publication was inevitable, but this time lag should not bias the findings because no evidence exists to suggest that studies published before 2020 would find different outcomes from those published in 2020 or in the first half of 2021. Finally, despite being a comprehensive review, data were still limited. Our trend estimates for associations between regain in weight and the content of programmes mainly relied on indirect comparisons; because comparisons were across rather than within trials in most instances, these comparisons were observational and could be confounded. Also, few studies lasted more than five years, meaning findings should not be extrapolated beyond this point. For some variables, relatively few studies contributed data, and hence the lack of associations seen should not be taken as evidence of absence.

\section{Comparisons with other studies}

Considerable research has been done on developing and testing specific strategies for maintaining weight loss, but these strategies are not regularly implemented in routine practice. Low intensity interventions have proven to be ineffective, ${ }^{10}$ whereas a systematic review of more intensive interventions providing ongoing behavioural support, in effect extending the behavioural weight loss programme, reported a reduction in regain in weight of $-1.56 \mathrm{~kg}$ (95\% confidence interval -2.27 
to -0.86$).^{5}$ This analysis included data from the weight loss phase, with most studies reporting overall weight loss, not regain. Moreover, attendance at behavioural weight loss programmes declines over time and many participants struggle to maintain the cognitive effort required to adhere to ongoing interventions. ${ }^{6}$ Some weight loss programmes included specific strategies for maintaining weight loss but we found no evidence that these strategies improved long term outcomes. Programmes that were available to participants outside of the clinical trial setting were associated with weight loss beyond the end of the programme, however. This finding is consistent with the results of a recent randomised controlled trial of a total diet replacement programme; 30 months after the end of the programme, participants who reported trying to lose weight in the last three months (of their own volition) maintained greater weight loss $(-7.6 \mathrm{~kg} v-4.8$ $\mathrm{kg}$ in the total diet replacement group; $-3.6 \mathrm{~kg} v-1.6$ $\mathrm{kg}$ in the usual care group). ${ }^{11}$ This result could suggest that focusing on recurrent weight loss interventions rather than maintenance of weight loss might be a more helpful public health strategy.

We found no evidence that a focus on diet, physical activity, or both, altered the rate of regain in weight, although a previous review suggested that behavioural weight loss programmes which involved both diet and physical activity were associated with greater weight loss than diet or activity alone. ${ }^{12}$ Evidence from one model indicated that structured programmes where the intensity of the intervention reduced over time rather than stopped abruptly were modestly more successful, perhaps because they help prepare participants to maintain their efforts without regular supervision; we are not aware of other studies that have directly explored this hypothesis. In contrast, programmes including financial incentives were associated with substantially greater regain in weight, which is likely to negate the benefit of adding this element to programmes within a year, and should be taken into account when considering providing these programmes. A previous systematic review noted the short term effectiveness of financial incentives for weight loss, but highlighted the need to explore ways to maintain weight loss in the longer term. ${ }^{13} \mathrm{~A}$ broader review which examined the use of financial incentives for a range of health related behaviours found that the benefits disappeared more than three months after the end of the programme. ${ }^{14}$ In contrast, some evidence indicates that when used to stop smoking, the benefits of financial incentive programmes persist, perhaps because of differences in the behavioural processes involved in stopping smoking compared with changes in an ongoing behaviour, such as eating or activity. ${ }^{15}$

Two previous systematic reviews suggested that very low energy diets, typically based on total meal replacement interventions and use of partial meal replacements, result in greater weight loss than comparator programmes. ${ }^{1617}$ We showed that greater weight loss was associated with faster regain in weight, and unsurprisingly, in univariable analyses meal replacements were associated with greater regain in weight. We found that this association was reversed after adjustment for initial weight loss when all studies were included, similar to the trial that showed that the rate of regain in weight was determined by the amount of weight lost, not whether weight loss was achieved by meal replacement or standard dietary means. ${ }^{18}$ When studies at high risk of bias were excluded, however, the association between partial meal replacements and greater regain in weight was no longer explained by weight loss during the programme. This finding warrants further exploration, including potential differences between total and partial meal replacement, but our main analyses suggested that the commonly held view that meal replacement programmes produce rapid regain in weight because this approach is unsustainable might not be true.

\section{Policy implications}

Regain in weight after the end of a programme and the associated change in weight related comorbidities affects the cost effectiveness of behavioural weight loss programmes, but is often not measured. ${ }^{19}$ Estimates of the cost effectiveness of programmes are therefore usually based on assumptions about regain in weight rather than empirical data, resulting in inconsistencies. Our finding that greater initial weight loss was associated with faster regain in weight can help inform future modelling and projections. However, this finding should not be taken to imply that programmes achieving smaller weight losses are more effective in the longer term. Rather, our results imply that greater weight loss during the programme should preserve a greater difference between the intervention and comparator groups for at least five years. Thus, compared with interventions for maintaining weight loss, achieving greater initial weight loss is currently the most effective strategy to achieve sustained improvements in cardiovascular health in the long term (based on unpublished data). Although the differences between programmes were modest, some weight loss programmes can produce substantial differences in weight loss at the end of the programme; a recent trial of total diet replacement found a $7 \mathrm{~kg}$ difference compared with a standard behavioural weight loss programme. ${ }^{20}$ A difference in body mass index of one is associated with a $7 \%$ difference in mortality, mostly from cardiovascular disease. ${ }^{21}$

\section{Conclusions}

Obesity is considered a chronic relapsing condition and, in general, when an intervention is stopped, participants who follow these weight loss programmes regain weight. ${ }^{22}$ Our data showed that regain in weight after weight loss in a weight management programme was faster with greater initial weight loss, but it takes an average of five years to remove the weight advantage (and thereby the beneficial effect on cardiometabolic risk) that greater initial weight loss achieves (fig 2). Such legacy benefits for ongoing, although declining, reduction in risk factors for cardiovascular disease 
have been shown to improve major clinical outcomes in studies of interventions for hypertension and high lipid levels. ${ }^{23-25}$ We found no evidence that providing support for specific maintenance of weight loss during the programme reduced the regain in weight. Instead, programmes that are widely available to allow individuals to re-engage when they want to might be the best strategy for supporting longer term lower rates of change in weight.

\section{AUTHOR AFFILIATIONS}

${ }^{1}$ Nuffield Department of Primary Care Health Sciences, University of Oxford, Oxford, UK

${ }^{2}$ NIHR Oxford Biomedical Research Centre, Oxford University Hospitals NHS Foundation Trust, Oxford, UK

3 Oxford Biomedical Research Centre, Nuffield Department of Population Health, University of Oxford, Big Data Institute, Oxford,

${ }^{4}$ Division of Epidemiology and Public Health, School of Medicine, University of Nottingham, Nottingham, UK

${ }^{5}$ Royal Free Hospital, Royal Free London NHS Foundation Trust, London, UK

${ }^{6}$ Nuffield Department of Primary Care Health Sciences, University of Oxford, Oxford, UK

${ }^{7}$ United Nations World Food Programme, Cox's Bazar, Bangladesh

${ }^{8}$ Faculty of Medical Sciences, Population Health Sciences Institute, Newcastle University, Newcastle upon Tyne, UK

${ }^{9}$ Faculty of Behavioural, Management, and Social Sciences, University of Twente, Enschede, Netherlands

We thank the people with overweight and obesity who shaped this work at the outset, and Anna Whiting and Philippa Seeber for serving as patient and public involvement advisors on this work. Nia Roberts, subject librarian, designed and executed searches. Alison Avenell, Brian Taylor, and colleagues at Aberdeen provided invaluable support and advice throughout. Initial work on database construction was funded by National Institute for Health Research (NIHR) HTA grant 15/09/04 - (REBALANCE) REview of Behaviour And Lifestyle interventions for severe obesity: AN evidenCE synthesis. We thank Sarah King, Mary Logan, and Yolanda Warren for their help with study screening. We thank Alex Robinson and Louis Robinson for their help with data entry. We thank Helen Parretti, Stephan Dombrowski, Alison Avenell, and Nerys Astbury for sharing data extraction forms from previous reviews. We are grateful to the many authors who answered our queries and provided additional data for our analyses.

Contributors: JH-B, PA, SAJ, FFS, FDRH, PS, and JLO conceived and designed the review. JH-B, RB, AT, ARB, AB, and AD conducted the screening. IH-B, AT, ARB, AB, and AD conducted the data extraction and assessed studies for risk of bias. JLO conducted the main statistical analyses. JH-B prepared the first draft of the review, with further input from AT, PA, and SAJ, and all authors contributed to the interpretation and final write up. All authors had direct access to the data, or access was provided as requested. JH-B is the guarantor. SAJ and PA are joint senior authors. The corresponding author attests that all listed authors meet authorship criteria and that no others meeting the criteria have been omitted.

Funding: This research was funded by the British Heart Foundation (BHF), PG/17/68/33247, and National Institute for Health Research (NIHR) Oxford Biomedical Research Centre (BRC) Obesity, Diet, and Lifestyle Theme. JH-B, PA, SAJ, and FDRH are part funded by NIHR Oxford BRC. JH-B is also part funded by an NIHR Cochrane Programme Grant. PA and SAJ are NIHR senior investigators and are also funded by NIHR Oxford and Thames Valley Applied Research Collaboration. FDRH also acknowledges part support from the NIHR School for Primary Care Research (SPCR), the NIHR Collaboration for Leadership in Applied Health Research and Care (CLAHRC) Oxford, and the NIHR Oxford BRC. The views expressed are those of the authors and not necessarily those of the BHF, NHS, NIHR, or the Department of Health and Social Care. The funders were not involved in study design, conduct, reporting, or decision to submit for publication.

Competing interests: All authors have completed the ICMJE uniform disclosure form at www.icmje.org/coi_disclosure.pdf and declare: support from the British Heart Foundation and National Institute for Health Research Oxford Biomedical Research Centre Obesity, Diet, and Lifestyle Theme for the submitted work. PA and SAJ were investigators on a trial of a low energy total diet replacement programme funded by Cambridge Weight Plan, and PA spoke at a seminar at a Royal College of General Practitioners' conference funded by Novo Nordisk-neither of these led to personal payments. All other authors have nothing to declare.

Ethical approval: Not required.

Data sharing: Data are from published research and therefore are mostly in the public domain. Extracted data are available on request from the corresponding author (jamie.hartmann-boyce@phc.ox.ac. uk)

The lead author affirms that the manuscript is an honest, accurate, and transparent account of the study being reported; that no important aspects of the study have been omitted; and that any discrepancies from the study as planned (and, if relevant, registered) have been explained; the protocol was registered in advance and all changes were detailed to the protocol record with reasons given. Dissemination to participants and related patient and public communities: Results will be shared by a briefing document developed with patient and public involvement representatives.

Provenance and peer review: Not commissioned; externally peer reviewed

This is an Open Access article distributed in accordance with the Creative Commons Attribution Non Commercial (CC BY-NC 4.0) license, which permits others to distribute, remix, adapt, build upon this work non-commercially, and license their derivative works on different terms, provided the original work is properly cited and the use is noncommercial. See: http://creativecommons.org/licenses/by-nc/4.0/.

1 Di Angelantonio E, Bhupathiraju ShN, Wormser D, et al, Global BM Mortality Collaboration. Body-mass index and all-cause mortality: individual-participant-data meta-analysis of 239 prospective studies in four continents. Lancet 2016;388:776-86. doi:10.1016/S01406736(16)30175-1

2 Warr W, Aveyard P, Albury C, et al. A systematic review and thematic synthesis of qualitative studies exploring GPs' and nurses' perspectives on discussing weight with patients with overweight and obesity in primary care. Obes Rev 2021;22:e13151. doi:10.1111/obr.13151

3 Raben A, Vestentoft PS, Brand-Miller J, et al. The PREVIEW intervention study: Results from a 3-year randomized $2 \times 2$ factorial multinational trial investigating the role of protein, glycaemic index and physical activity for prevention of type 2 diabetes. Diabetes Obes Metab 2021:23:324-37. doi:10.1111/dom.14219

4 Lean M, Hankey C. Keeping it off: the challenge of weight-loss maintenance. Lancet Diabetes Endocrinol 2018;6:681-3. doi:10.1016/S2213-8587(17)30405-9

5 Dombrowski SU, Knittle K, Avenell A, Araúio-Soares V, Sniehotta FF. Long term maintenance of weight loss with non-surgical interventions in obese adults: systematic review and meta-analyses of randomised controlled trials. BMI 2014:348.g2646. doi:10.1136/bmi.g2646

6 Ahern AL, Wheeler GM, Aveyard P, et al. Extended and standard duration weight-loss programme referrals for adults in primary care (WRAP): a randomised controlled trial. Lancet 2017:389:2214-25. doi:10.1016/S0140-6736(17)30647-5

7 Hartmann-Boyce J, Aveyard P, Hobbs F, et al. Systematic review of weight regain after behavioural weight management programmes and the impact on cardiometabolic risk factors, health outcomes, and quality of life [Protocol] CRD42018105744: PROSPERO; 2018. https:// www.crd.york.ac.uk/prospero/display_record.php?RecordlD=105744.

8 Higgins I. Cochrane handbook for systematic reviews of interventions. Version 5.1.0. Cochrane Collaboration, 2011.

9 Dansinger ML, Tatsioni A, Wong JB, Chung M, Balk EM. Meta-analysis the effect of dietary counseling for weight loss. Ann Intern Med 2007:147:41-50. doi:10.7326/0003-4819-147-1-200707030 00007

10 Daley A, Jolly K, Madigan C, et al. A brief behavioural intervention to promote regular self-weighing to prevent weight regain after weight loss: a RCT. Public Health Research 2019;7. doi:10.3310/phr07070

11 Astbury NM, Edwards RM, Ghebretinsea F, et al. Extended follow-up of a short total diet replacement programme: results of the Doctor Referral of Overweight People to Low Energy total diet replacement Treatment (DROPLET) randomised controlled trial at 3 years. Int) Obes (Lond) 2021. doi:10.1038/s41366-021-00915-1

12 Johns DJ, Hartmann-Boyce J, Jebb SA, Aveyard P, Behavioural Weight Management Review Group. Diet or exercise interventions vs combined behavioral weight management programs: a systematic review and meta-analysis of direct comparisons. / Acad Nutr Diet 2014;114:1557-68. doi:10.1016/j.jand.2014.07.005

13 Ananthapavan J, Peterson A, Sacks G. Paying people to lose weight: the effectiveness of financial incentives provided by health insurers for the prevention and management of overweight and obesity - a systematic review. Obes Rev 2018;19:605-13. doi:10.1111/obr.12657 
14 Mantzari E, Vogt F, Shemilt I, Wei Y, Higgins JP, Marteau TM. Personal financial incentives for changing habitual health-related behaviors: A systematic review and meta-analysis. Prev Med 2015;75:75-85. doi:10.1016/j.ypmed.2015.03.001

15 Notley C, Gentry S, Livingstone-Banks J, Bauld L, Perera R, HartmannBoyce J. Incentives for smoking cessation. Cochrane Database Syst Rev 2019;7:CD004307. doi:10.1002/14651858.CD004307.pub6

16 Parretti HM, Jebb SA, Johns DJ, Lewis AL, Christian-Brown AM, Aveyard $P$. Clinical effectiveness of very-low-energy diets in the management of weight loss: a systematic review and meta-analysis of randomized controlled trials. Obes Rev 2016;17:225-34. doi:10.1111/ obr.12366

17 Astbury NM, Piernas C, Hartmann-Boyce J, Lapworth S, Aveyard P, Jebb SA. A systematic review and meta-analysis of the effectiveness of meal replacements for weight loss. Obes Rev 2019;20:569-87. doi:10.1111/obr.12816

18 Purcell K, Sumithran P, Prendergast LA, Bouniu CJ, Delbridge E, Proietto J. The effect of rate of weight loss on long-term weight management: a randomised controlled trial. Lancet Diabetes Endocrinol 2014;2:954-62. doi:10.1016/S2213-8587(14)70200-1

19 NICE. Weight management: lifestyle services for overweight and obese adults [PH53] 2014. https://www.nice.org.uk/guidance/ph53

20 Astbury NM, Aveyard P, Nickless A, et al. Doctor Referral of Overweight People to Low Energy total diet replacement
Treatment (DROPLET): pragmatic randomised controlled trial. BMJ 2018;362:k3760. doi:10.1136/bmj.k3760

21 Di Angelantonio E, Bhupathiraju ShN, Wormser D, et al, Global BM Mortality Collaboration. Body-mass index and all-cause mortality: individual-participant-data meta-analysis of 239 prospective studies in four continents Lancet 2016:388:776-86 doi:10.1016/S01406736(16)30175-1

22 Bray GA, Kim KK, Wilding JPH, World Obesity Federation. Obesity: a chronic relapsing progressive disease process. A position statement of the World Obesity Federation. Obes Rev 2017;18:715-23. doi:10.1111/obr.12551

23 Gallo G, Battistoni A, Coluccia R, Tocci G, Volpe M. Legacy effect in the treatment of hypertension: persistent cardiovascular protection after conclusion of randomized clinical trials in hypertension. Curr Hypertens Rep 2019;21:85. doi:10.1007/s11906-019-0991-2

$24 \mathrm{Ho}$ CLB, Sanders S, Breslin M, et al. Legacy effect of delayed blood pressure lowering drug treatment in middle-aged adults with mildly elevated blood pressure: systematic review and meta-analysis. / Hum Hypertens 2020;34:261-70. doi:10.1038/s41371-020-0323-7

25 Viñas Esmel E, Naval Álvarez J, Sacanella Meseguer E. The legacy effect in the prevention of cardiovascular disease. Nutrients 2020:12:E3227. doi:10.3390/nu12113227

Web appendix: Supplementary material 